\title{
Comunicación intercultural como medio para favorecer el cuidado culturalmente aceptable
}

\section{Intercultural communication as a means to promote culturally appropriate care}

Liliana González Juárez•

Ana Lucía Noreña Peña••

\section{Resumen}

Introducción: Un elemento central para el cumplimiento de la competencia cultural, que nos permita entender y desarrollar habilidades de comunicación con grupos culturales diversos, es ampliar nuestras habilidades en la comunicación intercultural. La comunicación intercultural favorece la calidad de atención de los servicios de salud. El objetivo del documento es describir la importancia de mantener una comunicación intercultural como medio para favorecer las prácticas de cuidado culturalmente competentes. Desarrollo: La cultura proporciona un significado heterogéneo al lenguaje de cada persona y grupo social. Es necesaria una cierta competencia comunicativa y no sólo una competencia lingüística. Es a través de la cultura donde se enseña a los individuos cómo comunicar a través del lenguaje, los gestos, los vestidos, la comida, la forma de utilizar el espacio, etc.. El etnocentrismo y el choque cultural, podría aflorar emociones negativas, por lo que se requiere de hacer un esfuerzo no solo de aproximarse a otras culturas sino repensar la cultura propia. Conclusiones: Evidentemente es necesario para proporcionar un cuidado culturalmente aceptable, estar interesado por conocer e integrarse a otras culturas, se proponen algunas estrategias como: la relación de ayuda, así como la participación de mediadores interculturales para lograr una mejor comprensión de los cuidados y tratamientos aplicados por el personal sanitario.

-Profesor titular "A" Escuela Nacional de Enfermería y Obstetricia de la Universidad Nacional Autónoma de México. Doctoranda de Enfermería y Cultura de los cuidados. Universidad de Alicante, España.

•.Profesor de enfermería. Escuela Universitaria de Enfermería. Universidad de Alicante, España. Correspondencia: 1lilil_24@yahoo.com.mx

RECIBIDO: 28 DE OCTUBRE DE 2010

ENVIADO: 15 DE NOVIEMBRE DE 2010

ACEPTADO: 10 DE DICIEMBRE DE 2010 


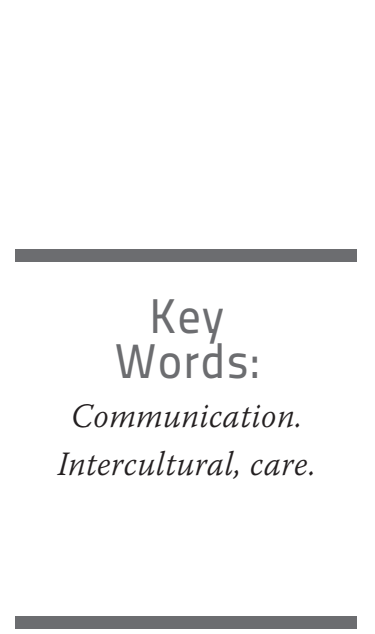

Abstract

Introduction: A central element for the implementation of cultural competence that allows us to understand and develop communication skills with diverse cultural groups, is to expand our skills in intercultural communication. Intercultural communication improves the quality of care health services. The document aims to describe the importance of intercultural communication as a means to encourage culturally competent care practices. Development: Culture provides a heterogeneous meaning to the language of every person and social group. We need a certain communicative competence and not only linguistic competence. It is through culture that teaches individuals how to communicate through language, gestures, clothing, food, how to use the space, etc. Ethnocentrism and culture shock, negative emotions could surface, for what it takes to make an effort not only to approach other cultures but to rethink the culture. Conclusions: obviously it is necessary to provide culturally acceptable care, be interested to know and integrate other cultures, suggests some strategies, like the aid relationship, as well as the participation of intercultural mediators to achieve a better understanding of the care and treatment applied by health personnel.

\section{INTRODUCCIÓN}

En respuesta a la globalización económica, el incremento de los medios de comunicación, los intercambios comerciales, la oferta de programas educativos, movimientos migratorios y mestizaje entre otros factores, se ha dado la conformación de comunidades multiculturales, donde a pesar de compartir un mismo espacio geográfico de convivencia, la integración entre los diferentes grupos étnicos no siempre es favorable.

En el ámbito de la salud se encuentra poco investigado la respuesta de la población, en torno a el uso de servicios de salud ante la diversidad cultural, algunos estudios refieren dificultades para la comprensión del lenguaje, así como para entender la organización de los servicios, asociadas al modelo biomédico prevaleciente.

Ante tal incremento de la diversidad cultural, la Organización
Mundial de la Salud, ha establecido un acuerdo para el logro de la denominada competencia cultural descrita por la Asociación Americana de Enfermería como: tener conocimiento, entendimiento y habilidades sobre grupos culturales diversos que permitan al profesional de salud, proporcionar cuidado cultural aceptable. ${ }^{1}$

Inicialmente, la competencia cultural exige por parte del personal sanitario, poseer cultura general, así como información culturalmente específica sobre los grupos étnicos, de tal manera que el profesional sepa qué preguntas hacer ${ }^{1}$ y como tratar a estos colectivos durante su atención. Por lo tanto, consideramos como elemento central para el cumplimiento de la competencia cultural, el ampliar nuestras habilidades respecto a la comunicación intercultural.
La comunicación intercultural se refiere a los contactos entre personas de diferentes culturas, las cuales se reúnen, interactúan e intentan resolver los problemas que se presentan en el marco las relaciones interpersonales. ${ }^{2}$ El logro de una comunicación intercultural, facilitará al personal sanitario garantizar la calidad de los servicios de salud otorgados, en el marco de un cuidado culturalmente aceptable.

La literatura muestra que la comunicación intercultural es una herramienta valiosa, y de acuerdo a nuestra experiencia, podría contribuir a mejorar la calidad de atención de los servicios de salud, ya que como refiere Cibanal, ${ }^{3}$ aun hablando de lo mismo, se comprende diferente, por lo tanto, es labor del personal sanitario facilitar la comprensión y el entendimiento de los 
tratamientos en el cuidado de las personas.

Los cuidados son considerados; actos donde cualquier persona ayuda a otra a asegurarle todo lo necesario para continuar su vida, en relación con la vida del grupo. ${ }^{4} \mathrm{Si}$ la comunicación es el medio, a través del cual se conciertan las prácticas de cuidado, el lograr una comunicación intercultural garantizará la subsistencia de los grupos humanos, ya que cada cultura aporta al lenguaje, una connotación diferente.

Por lo anterior, el objetivo del documento es describir la importancia de mantener una comunicación intercultural como medio para favorecer las prácticas de cuidado culturalmente competentes. Lo que inicialmente conlleva un cambio en las formas de comunicación y en la organización de los servicios de salud.

\section{LENGUAJE Y CULTURA}

La realización de prácticas de cuidado, da pauta a un indeterminado número de interacciones comunicativas, donde dos elementos sustanciales son, el lenguaje y la cultura, los cuales proporcionan un significado heterogéneo al lenguaje de cada persona y grupo social. Esto se puede apreciar en la diversidad de significados de salud y enfermedad que tiene cada grupo étnico ${ }^{5,6}$ y la falta de comprensión de estos conceptos, podría interferir en el proceso de recuperación de la salud de las personas.

\section{Connotación COMÚN}

La comprensión de las interacciones comunicativas, debe precisarse entre las personas implicadas en una situación de cuidado, a fin de entender y contextualizar las dife- rencias. Es claro que el uso de un lenguaje y connotación común en una situación de cuidado, inicialmente permitirá planear conjuntamente los cuidados culturalmente aceptables y lograr el entendimiento y aceptación de los tratamientos establecidos. Además, al conocer las características étnicas y culturales de cada grupo, se determina tanto el tipo y significado del lenguaje verbal como el no verbal, pues hablando la misma lengua se comprende diferente.

Algunos estudios en población inmigrante, revelaron que este colectivo enfrenta dificultades en el acceso a los servicios sanitarios, debido a los problemas en el dominio del lenguaje, la falta de familiaridad con la organización de los servicios sanitarios y el estado socio-económico bajo. ${ }^{7,8}$ Otros enfatizan, que los desacuerdos son atribuidos a la discrepancia entre la visión cultural y la falta de familiaridad con la medicina occidental. ${ }^{9,10,7}$

Es necesaria una cierta competencia comunicativa y no sólo una competencia lingüística. Por un lado, la comunicación verbal es muy importante, y por otro, no se puede presuponer que los sistemas de comunicación de otras culturas sean semejantes a los -autóctonos-. ${ }^{11}$ Por tal motivo, los servicios de salud deben ser sensibles y accesibles a las necesidades de los diferentes grupos étnicos prevalecientes.

\section{Desconocimiento DE LA CULTURA DEL OTRO}

Es a través de la cultura donde se enseña a los individuos cómo comunicar a través del lenguaje, los gestos, los vestidos, la comida, la forma de utilizar el espacio, etc. ${ }^{3}$
En verdad, el contexto cultural se encuentra cargado de significados tanto para la persona como para la familia y los grupos sociales.

Watzlawick, ${ }^{12}$ refiere que un fenómeno permanece inexplicable en tanto el margen de observación no es suficientemente amplio como para incluir el contexto en el que dicho fenómeno tiene lugar, sin embargo en los servicios de salud comúnmente puede apreciarse una comunicación asimétrica entre el personal y población usuaria de los servicios, lo que se contrapone a la comunicación intercultural donde se requiere establecer una situación de mayor igualdad posible.

El proceder de un grupo cultural diferente de quien otorga los cuidados, puede actuar como barrera en el proceso de atención. Las interacciones comunicativas con la persona sujeto de cuidado, pueden verse limitadas por problemas de comprensión del lenguaje, desconocimiento de la cultura del otro o bien por una insuficiente consciencia de la propia cultura. ${ }^{13}$

Cuando el proceso de atención irrumpa en lo más íntimo de la persona y su entorno sin prestar atención a los valores circundantes al fenómeno de enfermar/sanar, está perdiendo la mayor parte de su capacidad terapéutica. ${ }^{14}$ Es aquí donde el conocimiento y reconocimiento de la cultura de salud del receptor de cuidado podrían contribuir a explicar o entender la situación de salud.

El grupo social donde formamos parte, dota a la persona de diferente tipo de referentes de donde se desprenden los valores, creencias y normas sociales de la cultura. ${ }^{15}$ De esta manera, las personas que llegan a formar parte de otros 
grupos culturales, se encuentran en una situación de desigualdad, mientras no logren una adaptación a las prácticas de cuidado cotidiano del nuevo grupo o bien sean acogidos por grupos que faciliten su inserción.

En la realización de los cuidados el personal sanitario, puede enfrentarse con personas procedentes de culturas diferentes a la suya, lo cual puede repercutir en una confrontación con sus propios valores y creencias, así como en el trato que proveerá a esas personas. Lo que caracteriza, la mayoría de las veces, a una deficiente comunicación intercultural es el desconocimiento que se tiene sobre la otra cultura. ${ }^{13}$ $\mathrm{Y}$ frecuentemente se invierte poco tiempo en la valoración de los aspectos socioculturales que faciliten el cuidado a la salud.

Se puede tener la idea, de que una persona con diferente origen cultural, es portadora de enfermedades infectocontagiosas, al respecto algunos estudios muestran que no es así16 y por el contrario, un aislamiento social podría deteriorar la salud de estos grupos o bien llegar a producir alienación o segregación de ciertas personas o grupos étnicos.

\section{EtNocentrismo}

Es esencial evitar el etnocentrismo, comprendido como, la creencia que los propios valores, creencias y maneras de conocer y hacer son las mejores, o superiores a otras. Los problemas llegan cuando estas creencias son muy fuertes y controlan de manera no deseable a otros que son culturalmente diferentes. ${ }^{17}$ Esta situación nos puede llevar a un choque cultural y a un enfrentamiento innecesario con la persona y su familia, donde se diversifican las dudas y se dilata la recuperación del paciente.

El choque cultural, no sólo implica una incomprensión del comportamiento ajeno, sino que también afloran una serie de emociones negativas: desconfianza, incomodidad, ansiedad, preocupación y violencia. Para superar esto hay que comunicarse ${ }^{13}$ interculturalmente y emocionalmente. Lo cual implica un conocimiento del contexto, lengua, comunicación verbal y no verbal, creencias, valores y sentimientos de la persona.

Un estudio realizado en España, refiere que para la población inmigrante existen varios aspectos de la sociedad de acogida que son fuentes comunes de dificultad para el ajuste socio-cultural algunos de ellos son: la percepción de una mayor planificación de la vida económica y social; una mayor igualdad tanto general como en las relaciones entre géneros; y un grado más alto de la cultura local suponen un esfuerzo de ajuste para todos los grupos, ${ }^{18}$ en el proceso de integración a la sociedad de acogida y al uso de servicios de sanitarios.

Realizar un autoanálisis objetivo de las circunstancias que motivan la presencia de diferentes grupos étnicos, en la sociedad de acogida, podría servir de base para la integración cultural. Con el propósito de no caer en la denigración de la cultura ajena para ensalzar la propia. $^{13}$

Es importante, ser consciente del punto de vista etnocentrista y empezar a repensar muchos de los valores de lo que hasta hora fundamentaban lo que se ha denominado $<<$ identidad cultural $>>$.

\section{Re-CONOCIMIENTO DE LA PROPIA CULTURA.}

La comunicación intercultural no sólo implica una aproximación a otras culturas, sino también el esfuerzo de repensar la cultura propia. En los contactos interculturales es muy frecuente utilizar el método comparativo para describir nuestra experiencia. Así se viene, a establecer lo que es común y lo que es distinto. ${ }^{13} \mathrm{Al}$ comparar se enfatiza aquello que se contrapone a nuestros propios valores y creencias. Si se parte del principio ético de que todas las culturas son dignas y merecedoras de respeto. ${ }^{13}$ Estaremos en una posición de igualdad frente a la persona quien recibe los cuidados.

No solo se requiere un conocimiento de la cultura de quien otorga la atención sino de quien la recibe, donde se analice la respuesta ante las diferencias ya que, al evaluar el comportamiento o las creencias de los receptores de cuidados, podrían confrontarnos con nuestros propios valores sobre la salud y enfermedad.

\section{Comunicación Libre DE PREJUICIOS}

Inicialmente, el cuidado de personas con diferente origen cultural, implica una disposición e interés del personal sanitario en conocer la otra cultura, donde un elemento básico es contar con un lenguaje común, libre de prejuicios. El prejuicio se da cuando, o bien la persona que juzga no entiende a la otra y a su cultura, o bien extrapola la experiencia sobre un individuo al resto de sus miembros de su grupo. ${ }^{19}$

Evidentemente es necesario estar interesado por conocer e integrarse a otras culturas. Este interés 
no es sólo el deseo etnocéntrico de reafirmar la propia cultura en relación al "exotismo" del otro. Durante la comunicación intercultrural, es importante considerar algunos factores tales como el lugar de procedencia, creencias sobre el origen del padecimiento, situación socioeconómica, características culturales del grupo y el tipo de comunicación prevaleciente.

\section{Relación de Ayuda}

El cuidado de la salud precisa de una relación de ayuda donde el personal sanitario capte las necesidades del paciente, con el fin de auxiliar a descubrir otras posibilidades de percibir, aceptar y hacer frente a su situación actual. ${ }^{3}$

Identificar el significado que tiene para la persona su padecimiento y la importancia de su recuperación. El logro de la comunicación intercultural, se plantea como un reto, pues las situaciones de cuidado, comúnmente implican el establecimiento de una relación empática con la persona y su familia.

Encontrarse en un grupo cultural diferente al de origen, enfrentando un padecimiento puede colocar a la persona en una situación de vulnerabilidad, repercutiendo así en una recuperación, o bien a una segregación o marginación del grupo cultural de acogida, lo que puede llevar a complicaciones en el estado de salud.

La expresión de los sentimientos es una de las labores terapéuticas que el personal de salud desarrolla durante la atención. Los sentimientos, deben ser encausados, integrados, de modo que no sean éstos los que rijan el comportamiento, sino que sea la persona quien, desplegando el mundo de sus valores, aproveche la energía que tienen los sentimientos y afronte las dificultades, sean de la índole que sean. ${ }^{20}$

Acudir a los servicios sanitarios, implica, de acuerdo a investigaciones recientes que, el paciente y su familia ingresan, en un lugar donde pierden el control de la situación, que pasa a manos de los profesionales que ahí trabajan, se plantean problemas de índole lingüístico y cultural ligados a hábitos e incluso a la concepción de la prestación sanitaria que se les ofrece. ${ }^{15}$

\section{Mediadores interculturales}

Adicionalmente al interés del personal sanitario por la comunicación intercultural, entre miembros de diferentes grupos sociales, ${ }^{16}$ el contexto debe propiciar una situación de igualdad entre ambas partes por lo que en ocasiones se recomienda la participación de los mediadores interculturales.

La participación de mediadores culturales no sólo es traducir las palabras, sino que va más allá, abarcando todos los aspectos de la comunicación no verbal (olor, gestos, movimientos corporales, silencios, titos, etc.), que culturalmente son claves. La comunicación no verbal, adquiere significados desde el origen cultural. ${ }^{16} \mathrm{Y}$ como refiere Watzlawick, ${ }^{12}$ aún el silencio comunica algo.

A pesar de que algunos estudios implícitamente reconocen, la participación de mediadores interculturales, debido a que facilitan la interpretación de los síntomas, permiten conocer los remedios y tratamientos que usan para promocionar y recuperar la salud definidos culturalmente, ${ }^{15}$ actualmente su participación no se encuentra reglamentada ni generalizada en las instituciones de salud.

La participación de los mediadores interculturales, puede contribuir en la comprensión de los cuidados y tratamientos recibidos tanto al paciente como a su familia, así como, profundizar en conocer información relevante relacionada con el cuidado. No obstante, es evidente que falta mayor comprensión sobre su participación y aceptación.

\section{Retos PaRa la COMUNicación INTERCULTURAL}

El personal sanitario, contendemos continuamente con la falta de tiempo para escuchar a los pacientes, conocemos el beneficio terapéutico de mantener una comunicación estrecha con ellos, permite disipar dudas respecto al desarrollo de los cuidados y aplicación de los tratamientos. Sin embargo, lidiamos con algunos factores que inciden en una comunicación deficiente en los servicios sanitarios, tienen que ver con los escasez de personal y el incremento de la demanda de los servicios.

En este orden, la evidencia de algunos estudios sobre calidad de los servicios de salud, muestra que la población asigna importancia, a ser escuchados durante su atención, ${ }^{21}$ y paradójicamente, no se promueve la comunicación intercultural, lo que contribuye, al aumento de discrepancias entre pacientes y proveedores sanitarios. ${ }^{22}$

Así se propone incorporar en la práctica profesional la comunicación intercultural como herramienta básica de la competencia cultural y de los cuidados culturalmente aceptables. Además de la presencia de los mediadores inter- 
culturales en los servicios sanitarios, con el propósito de mejorar la atención de estos colectivos. Es importante considerar que la tolerancia y el respeto al diferente, es una norma de convivencia y es un criterio de calidad de la atención a la salud. ${ }^{14}$

\section{Referencias Bibliográficas}

1 Giger-J,Davidhizar R,Purnell L,Harden JT,Phillips J, Strickland O. American Academy of Nursing Expert Panel Report: DEveloping Cultural Competence to Eliminate Health Disparities in Ethnic Minoritie and other Vulnerable Populations Journal of Transcultural Nursing 2007; 18(2): 95-102.

2 Rodrigo-Alsina M. La interculturalitat. Els estudis de comunicació intercultural. DCIDOB Afers Internacionals 1996;56 9-12.

3 Cibanal- J L. Técnicas de comunicación y relación de ayuda en ciencias de la salud. Madrid España: Elsevier; 2003.

4 Collière M F. Promover la vida. De la práctica de las mujeres cuidadoras a los cuidados de enfermería. Madrid: Ed. Interamericana McGraw Hill. 1993.

5 Amezcua M. Antropología de los cuidados. Enfermedad y padecimiento: significados del enfermar para la práctica de los cuidados. Cultura de los Cuidados 2000; 7 y 8: 60-7.

6 López P, Rodriguez M, Barragan F, Bouzan ML, Martinez J, AbdulJabar M, et al. Diferentes percepciones de la salud y enfermedad entre profesionales y usuarios de programas de educación sanitaria en hipertensión y diabetes. una visión antropológica. Cultura de los cuidados 2008; 23: 35-45.
7 O'Mahony JM, Donnelly TT. The influence of culture on immigrant women's mental health care experiences from the perspectives of health care providers. Issues Ment Health Nurs 2007; 28 (5): 453-71.

8 Ahmad F, Shik A, Vanza R, Cheuhg AM, George U, Stewart DE. Voices of South Asian women: inmigration and mental health. Women Health 2004;40 (4): 113-30.

9 Weerasinghe M. Connection between the meaning of health and interaction with health professionals: caring for immigrant women. Health Care Women Int 2007;28 (4): 309-28.

10 Stewart MJ, Neufeld A, Harrison MJ,Spitzer D,Hughes K, Makwarimba E. Inmigrat women family caregivers in Canada: implications for policies and programmes in health and social sectors. Health Soc Care Community 2006;14 (4): 329-40.

11 Rodrigo M. Cuestionamientos, características y miradas de la interculturalidad. Sphera Pública 2004; 004: 53-68.

12 Watzlawick P. Teoría de la comunicación humana. Ed. Herder. Barcelona, 2002, $264 \mathrm{p}$.

13 Rodrigo M. Comunicación intercultural Barcelona: Anthropos. Barcelona, 1999.

14 López M, Barra A, Nuñez D, Jofre S. Interculturalidad en los servicios de salud. Index de Enfermería 2003; 43: 50-4.

15 Ruiz P. Psicología social de la comunicación.Madrid: Ed. Psicología Pirámide. 2006.

16 Fernández MA. Fenómenos migratorios y su influencia en los cuidados transculturales en el marco hospitalario. Cultura de los cuidados 2006, X (20): 62-8.
17 Leininger M. Cuidar a los que son de culturas diferentes requiere del conocimiento y las aptitudes de la enfermería transcultural. Cultura de los cuidados 1999; (6): 5-12.

18 Zlobina A, Basabe N, \& Páez D. Adaptación de los inmigrantes extranjeros en España, superando el choque cultural. Migraciones 2004; 31(15):43-84.

19 Spector R ,Muñoz M. Las culturas de la Salud. Madrid: Prentice Hall; 2003.

20 Bermejo J C. Apuntes de Relación de Ayuda. Madrid: Ed. Cuadernos del centro de humanización de la salud.1998.

21.-González-Valentín M, Padin S, De Ramón E. Satisfacción del paciente con la atención de enfermería. Enfermería clínica 2005; 15 (3); 147-55.

22 Ramalle-Gómara E. Enfermeras y médicos no deben asumir que su percepción de necesidades de cuidados coincide con la de sus pacientes. Enferm Clin 2008;( 6), (18): 330-32. 